\title{
Fault detection in power transformers using random neural networks
}

\author{
Amrinder Kaur ${ }^{1}$, Yadwinder Singh Brar ${ }^{2}$, Leena G. ${ }^{3}$ \\ ${ }^{1,2}$ Department of Electrical Engineering, I.K.Gujral Punjab Technical University, India \\ ${ }^{3}$ Department of Electrical and Electronics Engineering, Manav Rachna International Institute \\ of Reserach \& Studies, India
}

\begin{tabular}{l} 
Article Info \\
\hline Article history: \\
Received Apr 4, 2018 \\
Revised Jul 5, 2018 \\
Accepted Aug 2, 2018 \\
\hline
\end{tabular}

Keywords:

Dissolved gas analysis (DGA)

Fault diagnosis

Power transformer

Random neural network (RNN)

\begin{abstract}
This paper discuss the application of artificial neural network-based algorithms to identify different types of faults in a power transformer, particularly using DGA (Dissolved Gas Analysis) test. The analysis of Random Neural Network (RNN) using Levenberg-Marquardt (LM) and Broyden-Fletcher-Goldfarb-Shanno (BFGS) algorithms has been done using the data of dissolved gases of power transformers collected from Punjab State Transmission Corporation Ltd.(PSTCL), Ludhiana, India. Sorting of the preprocessed data have been done using dimensionality reduction technique, i.e., principal component analysis. The sorted data is used as inputs to the Random Neural Networks (RNN) classifier. It has been seen from the results obtained that BFGS has better performance for the diagnosis of fault in transformer as compared to LM.
\end{abstract}

Copyright $(2019$ Institute of Advanced Engineering and Science. All rights reserved.

\section{Corresponding Author:}

Amrinder Kaur,

Department of Electrical Engineering,

I.K.Gujral Punjab Technical University

Jalandhar-Kapurthala Highway, Kapurthala, Punjab, India.

Email: kinguk75@gmail.com

\section{INTRODUCTION}

Power transformer is one of the important equipment in power system. The fault in transformer causes breakdown in power system which produces financial losses to power industry and inconvenience to the end user. In power transformers, liquid insulation in the form of mineral oil/transformer oil is being used as cooling agent. An impregnated insulation cellulose/paper is also used as solid insulation in transformer. Transformer oil as liquid insulation is very important as it provides electrical insulation, dissipates heat as cooling agent, protect the core \& winding and does isolation and moreover, prevent direct contact of atmospheric oxygen with winding.

Paper insulation of winding deteriorate with time of usage which results in deterioration of solid insulation [1], [2], [3]. The liquid insulation (transformer oil) when heated up due to working of transformer, decomposes and produce gases like hydrogen $\left(\mathrm{H}_{2}\right)$, methane $\left(\mathrm{CH}_{4}\right)$, acetylene $\left(\mathrm{C}_{2} \mathrm{H}_{2}\right)$, ethylene $\left(\mathrm{C}_{2} \mathrm{H}_{4}\right)$ and ethane $\left(\mathrm{C}_{2} \mathrm{H}_{6}\right)$. These gases deteriorate the quality of transformer oil and further its properties as coolant and insulator are affected which may result in breakdown of transformer as equipment in power supply. This can be prevented by knowing the amount of gases dissolved in the transformer oil at regular intervals of time of usage. The conventional methods like Roger's ratio method, Dornenburg's method, Duval's triangle method and key gas ratio methods are used to find the fault in respect of amount of harmful gases dissolved in the transformer oil. But, these methods sometimes give a false fault type [4], [5]. To improve these anomalies in conventional methods, various software based intelligent methods such as artificial neural networks [6], [7], [8], [9], [11], Wavelet Analysis, Least Vector Quotient, Probabilistic Neural Network (PNN), fuzzy logic, Support Vector Machine classifiers and Self-Organizing Map classifiers have been 
proposed to find faults in transformers, [12], [13], [14], [15], [16]. As this is diagnosis process so neural network can be applied successfully [18].

This paper proposes a new ANN (Artificial neural network) based algorithm Random Neural Network to find faults in power transformers using BFGS (Broyden-Fletcher-Goldfarb-Shanno) and LM (Levenberg-Marquardt). Further, linear regression and Bland Altman techniques are used to compare BFGS and LM algorithm. Also, Receiver Operating Characteristic (ROC) curve is used to validate the results. It has been found out that BFGS outperform the LM algorithm.

\section{FAULT CLASSIFICATION ALGORITHM APPROACH}

Following are the steps implemented for the proposed transformer fault classification scheme. The raw data are collected from PSTCL (Punjab State Transmission Corporation Ltd.) labs and preprocessed. After that feature selection is performed so that all faults are covered in the data selected and in the last step of the classification, ANN (Artificial neural network) based classifiers have been applied to determine different faults.

\section{PROBLEM FORMULATION}

\subsection{Dissolved gas analysis (DGA)}

The DGA (Dissolved Gas Analysis) is the most common techniques used for incipient fault diagnosis. Oil samples are collected to perform DGA and hence gas amount in the oil sample. The level of gases generated in oil-filled transformer provides the first level information for fault detection in transformer based on various conventional methods. Faults in oil-filled transformers can be found out according to the amount and type of gases generated These gases are hydrogen $\left(\mathrm{H}_{2}\right)$, methane $\left(\mathrm{CH}_{4}\right)$, ethylene $\left(\mathrm{C}_{2} \mathrm{H}_{4}\right)$, ethane $\left(\mathrm{C}_{2} \mathrm{H}_{6}\right)$, acetylene $\left(\mathrm{C}_{2} \mathrm{H}_{2}\right)$, carbon monoxide $(\mathrm{CO})$, and carbon dioxide $\left(\mathrm{CO}_{2}\right)$. Various conventional methods generally based on defined principles such as gas concentrations, key gases, key gas ratios, and graphical representations. Under IEEE Standard C57.104- 2008 Key Gas Analysis, Dornenberg and Rogers Ratio Methods, Nomograph, IEC Ratio, Duval Triangle, and CIGRE Method are listed to find out faults in transformers. The DGA can find faults such as partial discharge (corona), overheating, and arcing in many different power transformers. Like a blood test in human body, DGA can provide the early diagnosis to find incipient faults and increase the chance of finding an appropriate maintenance schedule or repair if required. Table 1 shows different faults of power transformer as given by IEC/IEEE. This will be used to train RNN neural network to find fault in transformers. Currently seven methods based on dissolved gas data are used to diagnosis types of faults:

a. Key Gas Method,

b. Dornenburg Ratio Method,

c. Rogers Ratio Method,

d. Nomograph Method,

e. IEC Ratio Method,

f. Duval Triangle Method and

g. CIGRE Method.

Table 1. Faults in Power Transformers

\begin{tabular}{clcc}
\hline S No & Type of Fault & Short Name & Code used for fault \\
\hline 1 & Partial discharge & PD & 0 \\
2 & Partial discharge with low energy density & D1 & 1 \\
3 & Partial discharge with High energy density & D2 & 2 \\
4 & Thermal fault with temp. less than $300^{\circ} \mathrm{C}$ & T1 & 3 \\
5 & Thermal fault with temp. between $300^{\circ} \mathrm{C}$ to $700^{\circ} \mathrm{C}$ & T2 & 4 \\
6 & Thermal fault with temp. greater than $700^{\circ} \mathrm{C}$ & T3 & 5 \\
\hline
\end{tabular}

\subsection{Data collection}

The gas samples of transformers from various substation of Punjab State Transmission Corporation Ltd (PSTCL), Ludhiana have been used as data for analysis. The data is collected as per the American Society for Testing and Materials (ASTM) standards. After the data collection, data is processed by removing linear trends, outliers, etc. Table 2 shows the data of samples obtained from PSTCL situated in Ludhiana.Total data collected from laboratory are 700 samples. After processing 600 samples are selected and100 sample per fault are used as input data. For reference only 5 samples are shown in Table 2. 


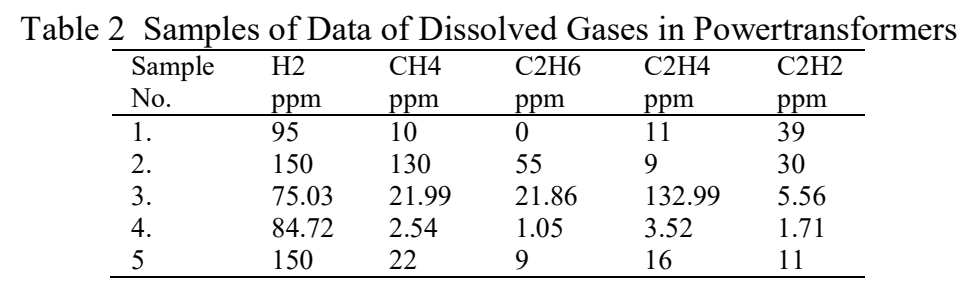

\section{RANDOM NUERAL NETWORK}

Random Neural Networks (RNNs) are a type of Artificial Neural Networks (ANNs) that could also be specified as type of queuing network. The information is presented as a dataset of labeled samples. The aim is "to learn" the relationship between input and output features. This learning process is done based on a set of examples in order to generate a learning model with the power of "generalising", this is to make "good" predictions for new unseen inputs. This procedure is based on the classical back propagation algorithm [11]. As in practice, the input and output variables in learning problems are bounded with known bounds, the algorithm described in [12] assumes that $\mathrm{a}(\mathrm{k}) \in[0 . .1] \mathrm{I}$ and $\mathrm{b}(\mathrm{k}) \in[0 . .1] \mathrm{O}$, for all sample $\mathrm{k}$. The RNN model as a predictor is a parametric mapping $\mathrm{v}\left(\mathrm{a}, \mathrm{w}^{+}, \mathrm{w}^{-}, \mathrm{L}\right)$, where the parameters $\mathrm{w}^{+} \mathrm{and}^{-} \mathrm{w}^{-}$ are adjusted minimizing the loss function. The network architecture is defined with I input nodes and $\mathrm{O}$ output nodes. There are no additional constraints regarding the network topology that means the network can be feedforward with one or several layers, or it can be recurrent network. We set the port of the input neurons each time that an input pattern a $(\mathrm{k})$ is offered to the network. The inputs to the positive ports are set with the input pattern: $\lambda+\mathrm{i}=\mathrm{a}(\mathrm{k}) \mathrm{i}$; the negative ports of input neurons are conventionally set to zero $(\lambda-\mathrm{i}=0)$. The output of the model is a vector of the activity rates produced by the output neurons. The adjustable parameters of the mapping are the weights connections among the neurons. RNN is implemented using two algorithm namely LM (Levenberg-Marquardt )and BFGS(Broyden-Fletcher-Goldfarb-Shanno) algorithms.

\section{RESULTS AND DISCUSSION}

In LM algorithm, at each epoch $\mu$, the approximation of the Hessian matrix is given by (1).

$$
H=H^{T} * H
$$

Where $H$ is hessian matrix. The dumping factor is modified at each epoch. In this case the calculated error E2 decreases, then the dumping factor $\eta$ is calculated by some constant value $\beta$ known as dumping constant $\alpha \leftarrow \eta / \beta$. Otherwise, the dumping value is increased by a factor of $\beta, \alpha \leftarrow \eta \beta$.

The 600 data samples collected from PSTCL lab were used for training the RNN with 100 samples for each fault. After 500 iterations error is reduced to 0.454 from 1038.21. The dumping constant for modifying the dumping factor which is taken as 3 , and $\beta=10$. The LM algorithm calculate the weight correction as

$$
\delta w=-G \times g
$$

where $g$ is gradient of input funcion and $\mathrm{G}$ is inverse of Hessian matrix. Then, the weights are updated by $\delta w$. Error plot as given in Figure 1.

BFGS (Broyden-Fletcher-Goldfarb-Shannon) Algorithm: The Broyden-Fletcher-Goldfarb-Shanno (BFGS) algorithm for the RNN model was proposed in 2000 by Likas and Stafylopatis [16]. It is an offline algorithm. At each epoch $\mu$ approximate Hessian matrix He $(\tau)$ is computed. The method starts with Positive definite Hessian matrix, then gradient and cost function are intialised from start point. The weight correction is calculated by using same equation (2) as used for Levenberg-Marquardt function. Gradient and cost function is calculated and change in gradient function is given by the equation.

$$
\delta g=g^{2}-g
$$

Using BFGS algorithm, after 500 iterations error is reduced to 0.166 from 1038.21. Error graph for BFGS is as shown in Figure 2. 


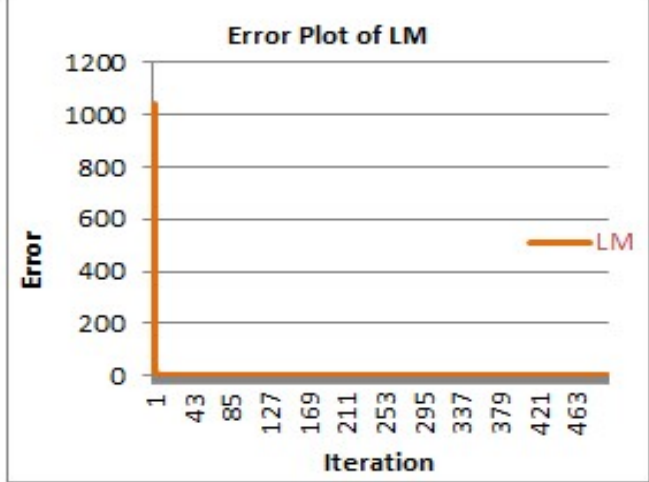

Figure 1. Error plot for LM algorithm

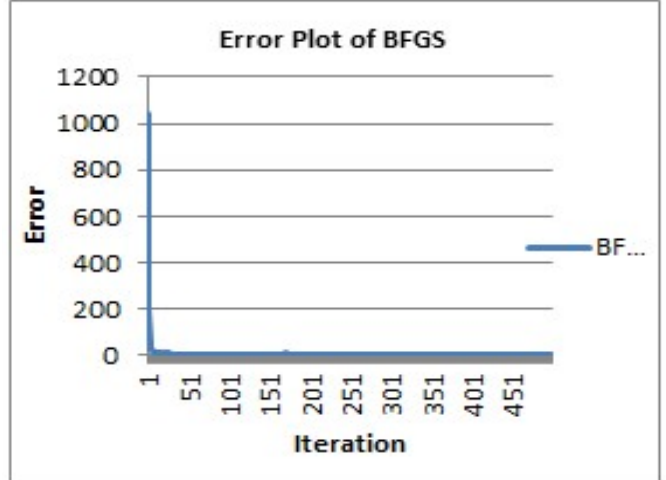

Figure 2. Error plot for BFGS algorithm

After training both the LM and BFGS algorithms were tested for 300 samples with 50 samples per fault. The linear regression and Bland Altman plot are used to compare clinical methods for diagnosis. In linear regression case plot between actual output and output from the proposed algorithm is plotted and gives coefficient of Determination $r^{2}$ and SSE denotes the Sum of square error [8]. It is an approach to model the relationship between actual output and output from the proposed algorithm. Regression minimizes the sum of squared differences from each point to the fitted line (or curve).

Bland-Altman plot [9] is a plot for matched pairs analysis which shows the relationship between the differences of actual and predicted output from proposed method versus the means of the these two outputs. RPC denotes reproducibility coefficient, CV is the coefficient of variation. Figure 3 and Figure 4 shows both the plots for LM and BFGS algorithms respectively.

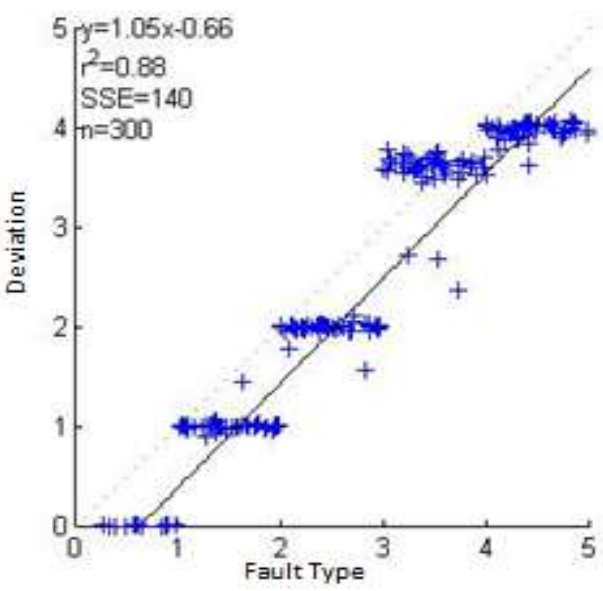

(a)

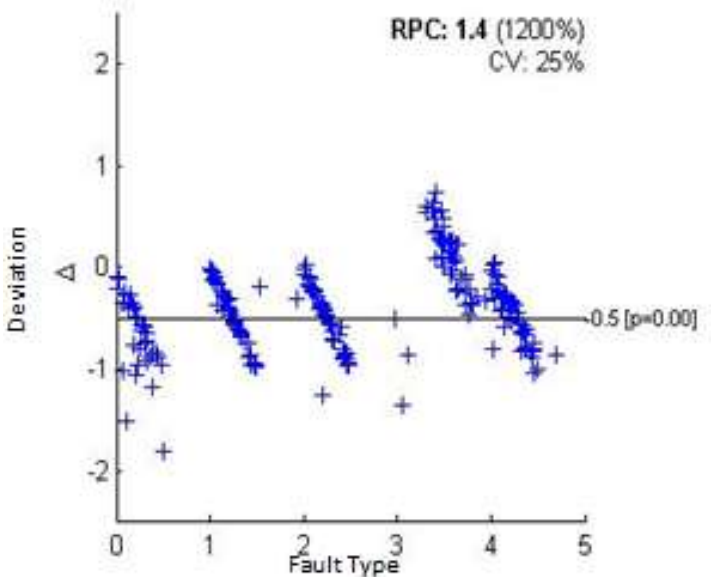

(b)

Figure 3. (a) Linear regression; (b) Bland altman plot of LM 


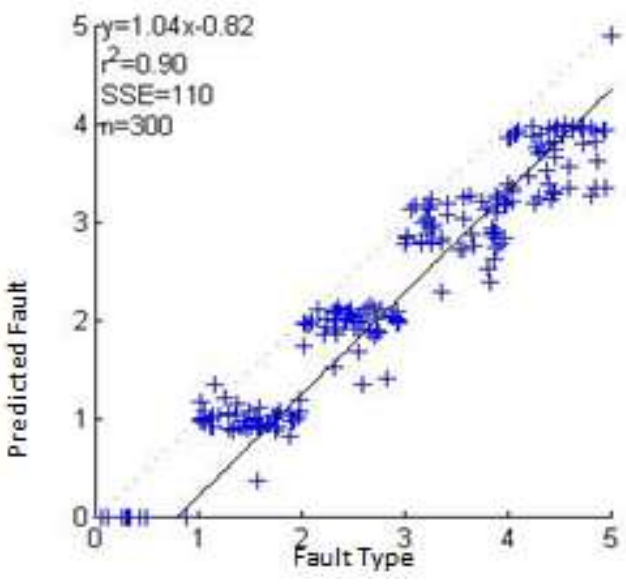

(a)

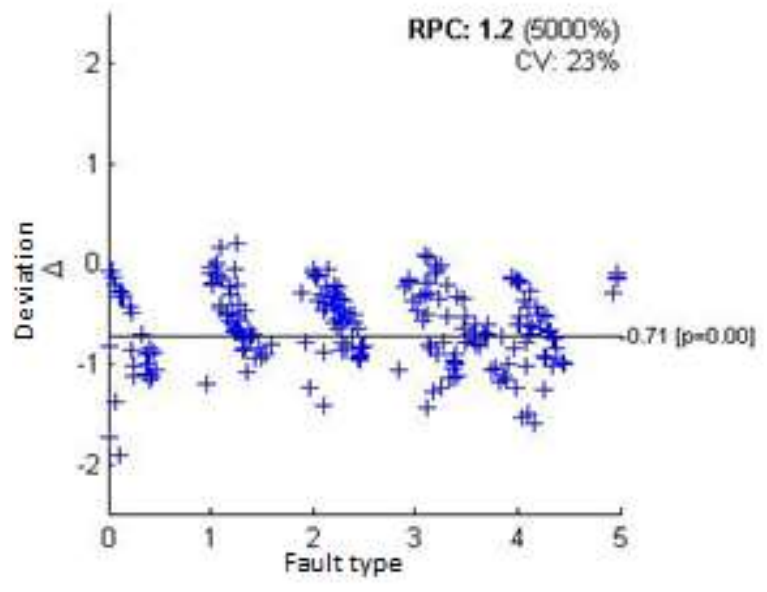

(b)

Figure 4. (a) Linear regression; (b) Bland altman plot of BFGS

A confusion matrix, also known as an error matrix is a specific table that is used to visualize the performance of an algorithm, it is usually called a matching matrix. Each row of the matrix represents the output by proposed algorithm while each column represents the actual output. Figure 5 and Figure 6 shows the confusion matrix for Random Neural Network to classify different transformer fault by using LM and BFGS algorithms. The accuracy obtained from these algorithms are $99.33 \%$ for BFGS and $94.66 \%$ for LM. These accuracies are $95.6 \%$ for Probabilistic Neural Network classifier and $93.6 \%$ for Backpropagation Network classifier (Levenberg-Marquardt Method) [5].

For further validation of results ROC curves [10] are plotted for both the algorithms and shown in Figure 7 and Figure 8. The Receiver Operating Characteristic (ROC) curve is a plot of the true positive rate against the false positive rate for the different possible cutpoints of a diagnostic test. AUC (Area under Curve) is equal to the probability that an algorithm will rank a randomly chosen positive instance higher than a randomly chosen negative one and is measure of test accuracy. Area under the ROC curve is $80.6 \%$ for LM algorithm while for BFGs it is $80.3 \%$. Table 3 shows the comparison of both algorithm based on sensitivity and specificity.

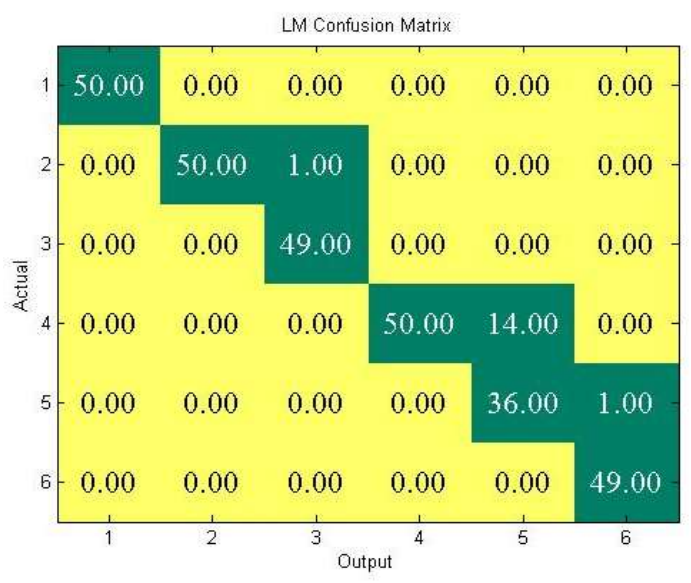

Figure 5. Confusion matrix for LM algorithm

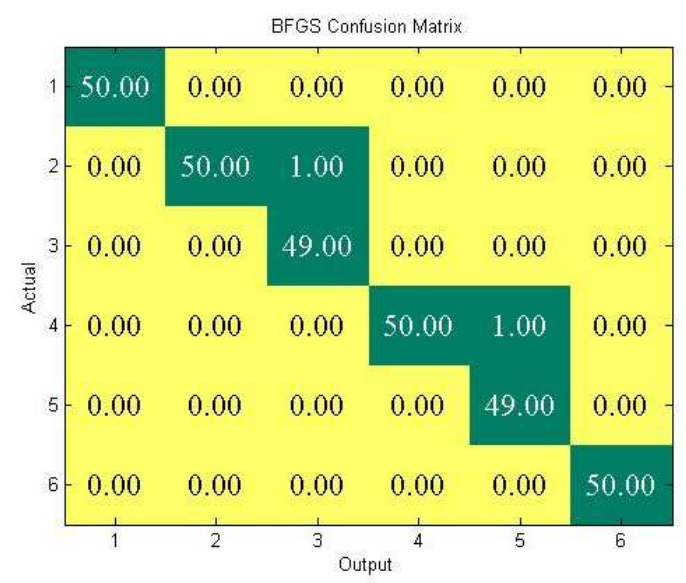

Figure 6. Confusion matrix for BFGS algorithm

Table 3. Comparison of BFGS and LM Training Algorithm for RNN

\begin{tabular}{cccc}
\hline Algorithm & Recognition Rate & Sensitivity & Specificity \\
\hline LM & $95 \%$ & 0.955 & 0.99 \\
BFGS & $99 \%$ & 0.993 & 1 \\
\hline
\end{tabular}




\section{ROCCurve / Test $/$ AUC $=0.806$}

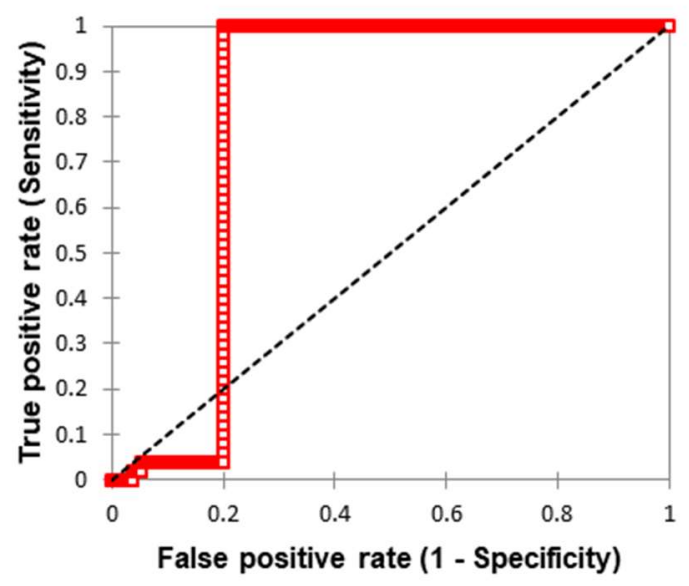

Figure 7. ROC for LM
ROC Curve / Test / AUC $=0.803$

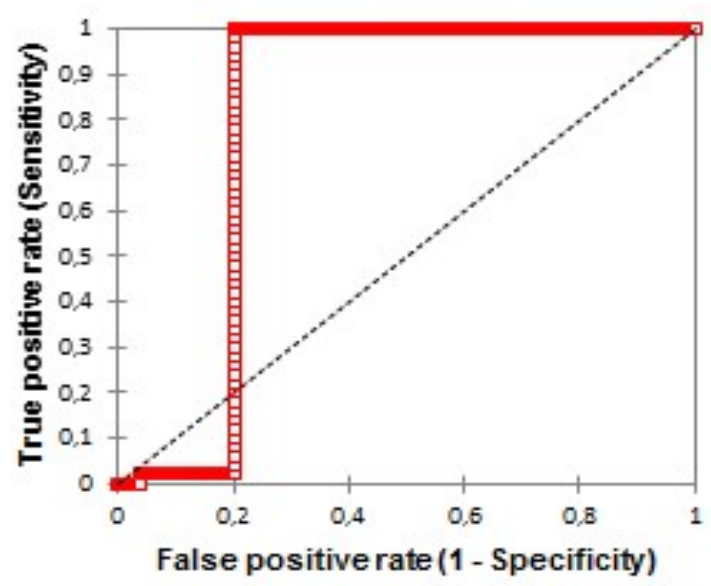

Figure 8. ROC for BFGS

\section{CONCLUSIONS}

RNN based Fault Diagnosis method (intelligent method based on AI techniques) is projected for fault recognition in Power Transformers. Based upon the results attained, it is concluded that finding/detection of faults in Power Transformers could be efficiently done using this intelligent method. In this paper, RNN is executed by using two different algorithms i.e. LM and BFGS. Both of these algorithms are compared by using various techniques like Regression Plots, Confusion Matrix and ROC Curve (proven methods to corroborate any diagnosis test/algorithm). It is also distinguished that results from BFGS is augmented in comparison to LM algorithm. Moreover, memory requirement for BFGS is also less as compared to LM algorithm as BFGS is a Quasi-Newton method, and will converge in fewer steps [19]. Hence, proposed RNN with BFGS algorithm could effectually be implemented to diagnose fault in Power Transformers.

\section{REFERENCES}

[1] Jayaswal P, Verma SN, Wadhwani AK, "Application of ANN, Fuzzy Logic and Wavelet Transform in Machine Fault Diagnosis Using Vibration Signal Analysis,” J Qual Maint Eng 16:Pp190-213, 2010.

[2] Verma P, Singh J, Sood YR, "The Influence of Service Aging on Transformer Insulating Oil Parameters," IEEE Trans Dielectr Electr Insul, 19:Pp421-426, 2012.

[3] Hasmat Malik, Amit Kumar Yadav, Sukumar Mishra, Tarkeshwar Mehto, "Application of Neuro-Fuzzy Scheme to Investigate the Winding Insulation Paper Deterioration In Oil-Immersed Power Transformer", Electrical Power and Energy Systems, 53, pp. 256-271, 2013.

[4] Knapp GM, Jovadpour R, His-Pin Wang, “An ARTMAP Neural Network: Based Machine Condition Monitoring System," J Qual Maint Eng, 6:Pp86-105, 2000.

[5] Tapsi Nagpal, Yadwinder Singh Brar, "Artificial Neural Network Approaches for Fault Classification: Comparison and Performance", Neural Computing and Applications, Volume 25 Issue 7-8, Pp 1863-1870, December 2014.

[6] Pampa Sinha, Sudipta Debath, Swapan Kumar Goswam, "Classification of Power Quality Events Using Wavelet analysis and Probabilistic neural Network", IAES International Journal of Artificial Intelligence (IJ-AI), Vol. 5, No. 1, pp. 1 12, 2016.

[7] Yadaiah N, Ravi N, "Internal Fault Detection Techniques Forpower Transformers" Application of Soft Computing, 11 Pp:5259-5269, 2011.

[8] Bhalla D, Bansal rk, Gupta HO, "Integrating AI based DGA fault diagnosis using Dempster-Shafer Theory," Int $J$ Electr Power Energy Syst, 48:Pp 31-38, 2013.

[9] Gardner MJ, Altman DG, "Calculating Confidence Intervals for Proportions and Their Differences". In: Gardner MJ, Altman DG, eds. Statistics with confidence. London: BMJ Publishing Group,:Pp28-33, 1989.

[10] Wen Zhu, Nancy Zeng, Ning Wang, "Sensitivity, Specificity, Accuracy, Associated Confidence Interval and ROC Analysis with Practical SAS ${ }^{\circledR}$ Implementations" NESUG, 2010.

[11] Chandrahas Mishra, D. L. Gupta, Deep Machine Learning and Neural Networks: An Overview," IAES International Journal of Artificial Intelligence (IJ-AI), Vol. 6, No. 2, pp. 66 73, 2017.

[12] Huo-ChingSun, Yann-Chang Huang, Chao-Ming Huang, " Fault Diagnosis of Power transformer Using Computational Intelligence: A Review”, Energy Procidia 14 (2012), Pp 1226 -1231, 2012. 
[13] E. Rumelhart, G. E. Hinton, and J. L. McClelland, "A General Framework for Parallel Distributed Processing. In Parallel Distributed Processing: Explorations in the Microstructure of Cognition," Volume 1 of Computational odels of Cognition and Perception, chapter 2,. MIT Press, Cambridge, MA, 1986b Pp45-76, 1986.

[14] Gelenbe, "Learning in the Recurrent Random Neural Network," Neural Computation, 5 (1):, 1993a. ISSN 08997667 Pp154-164, 1993.

[15] Parvin Darabad V, Vakilian M, Phung BT, Blackburn TR, “An Efficient Diagnosis Method For Data Mining On Single PD Pulses Of Transformer Insulation Defect Models," IEEE Trans Dielectr Electr Insul 20:Pp2061-2072, 2013.

[16] Liu J, Zheng K, Zhang H, Peng D, "A Comparative Research on Power Transformer Fault Diagnosis Based on Several Artificial Neural Networks," J Comput Inf Syst, 18:Pp7501-7508, 2013.

[17] Singh J, Sood YR, Jarial RK, "Condition Monitoring of Power Transformers-Bibliography Survey," IEEE Electr Insul Mag 24:Pp11-25, 2008.

[18] K. Chandana Rani, Y. Prasanth, “A Decision System for Predicting Diabetes using Neural Networks," IAES International Journal of Artificial Intelligence (IJ-AI), Vol. 6, No. 2, pp. 56 65, 2017.

[19] Likas and A. Stafylopatis, “Training the Random Neural Network using Quasi-Newton Methods,” Eur.J.Oper.Res, 126:Pp331-339, 2000.

\section{BIOGRAPHIES OF AUTHORS}
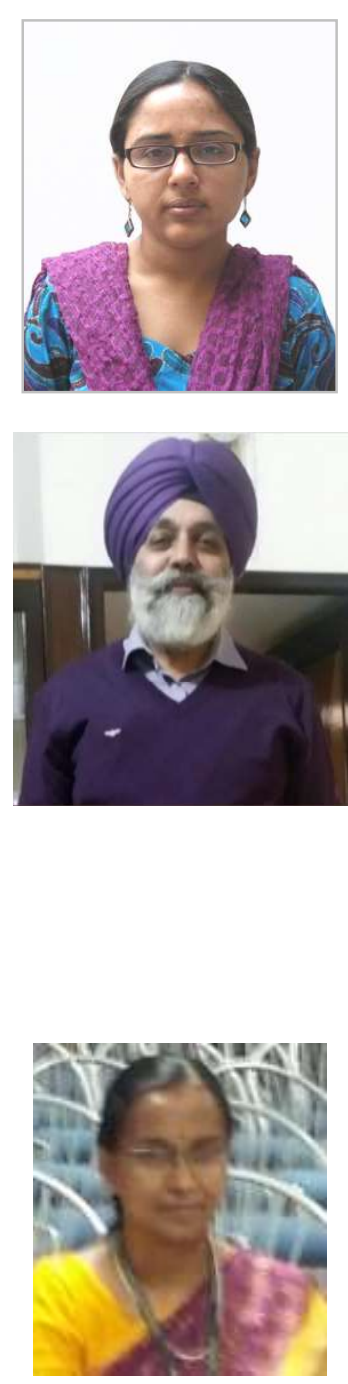

Amrinder Kaur received her B-Tech degree in Electrical Engg from Punjab Technical University in 2002 and M.Tech degree in Power Systems from YMCA University of Science \& Technology in 2013. She is pursuing her PHD from Punjab Technical University, India. She has more then 12 years of teaching/industrial experience and presently working as Asstt. Professor in the Department of Electrical and Electronics Engineering, Faculty of Engineering and Technology, of Manav Rachna International Institute of Reseach and Studies, Faridabad, India. Main areas of interest are Electrial Machines, Power System, Artificial Intelligence, Intelligent Control etc.

Y.S. Brar is working as Professor and Head in Department of Electrical Engineering at Inder Kumar Guzral .Punjab Technical University Kapurthala,. He obtained his B.E. (Electrical), M.E. (Power Systems) from Guru Nanak Dev Engineering College, Ludhiana and Ph.D. from Punjab Technical University, Jalandhar. He was involved in Teaching and development of several courses at Giani Zail Singh College of Engineering and Technology Bathinda. Dr Brar has served as Professor Department of Electrical Engineering and Dean Student Welfare at Guru Nanak Dev Engineering College Ludhiana. He has chaired various board of Studies at IKG Punjab Technical University Kapurthala, MRS Punjab Technical University Bathinda, SBS State Technical College Ferozepur etc. His research activities include Multi-objective power scheduling, Optimization techniques, Fuzzy theory applications in Engineering Problems. He has published/presented more than 100 papers in national and international journals/conferences. He has guided more than $08 \mathrm{Ph} . \mathrm{D}$ and more than $30 \mathrm{M}$.Tech students in his field. He has received 'Leading Educators of the World 2005' award from International Biographical Centre, Cambridge, England in the field of research, ISTE Best Teacher Award and Best Research Paper award.

Dr. Leena G. has B-Tech in Electrical and Electronics Engg. and M-Tech in Control Systems from Kerala University in 1991 and 1995 respectively. She completed her $\mathrm{PhD}$ in 2007 from Indian Institute of Technology, Kharagpur, India. She had over 20 years of teaching experience and presently she is Professor in the Department of Electrical and Electronics Engineering, Faculty of Engineering and Technology, of Manav Rachna International Institute of Reseach and Studies, Faridabad, India. Her areas of interest are nonlinear system, decentralized control, intelligent control, sliding mode control etc. 